\title{
Increases of Cardiometabolic Risk in Young Adults. Impact of Exercise Reductions during the College Years
}

\author{
Wolfgang Kemmler ${ }^{1^{*}}$, Michael Bebenek ${ }^{1}$, Simon Von Stengel ${ }^{1}$, Matthias Kohl ${ }^{2}$ \\ and Julia Bauer ${ }^{3}$ \\ ${ }^{1}$ Institute of Medical Physics, University of Erlangen-Nuremberg, Germany. \\ ${ }^{2}$ Department of Medical and Life Sciences, University of Furtwangen, Germany. \\ ${ }^{3}$ Institute of Dentistry, University Hospital Erlangen-Nuremberg, Germany.
}

\section{Authors' contributions}

All authors make substantial contributions to conception and design, the acquisition, analysis and interpretation of the data, the drafting and revision of the article and give approval to the final version.

Article Information

DOI: 10.9734/BJMMR/2015/17545 Editor(s):

(1) Gaetano Santulli, College of Physicians \& Surgeons, Columbia University Medical Center, New York, USA. Reviewers:

(1) Hiroyuki Tamemoto, Department of Biochemistry, Jichi Medical University, Japan (2) Paweł F. Nowak, Faculty of Physical Education and Physiotherapy, Opole University of Technology, Poland. (3) Ds sheriff, Faculty of Medicine, Benghazi University, Benghazi, Libya. Complete Peer review History: http://www.sciencedomain.org/review-history.php?iid=1118\&id=12\&aid=9162

\section{ABSTRACT}

Aims: The transition from school to university, traineeship or job especially along with moving away from home may lead to unhealthy lifestyle changes in young adults. Thus, we determined the effect of exercise changes during the college years on the development of cardiometabolic risk. Two cohorts of students with considerably varying demands on physical fitness (sport versus dentistry students) were compared.

Methodology: 53 randomly selected German male and female sport students (SPS, $20 \pm 2$ yrs.) and 61 male and female dental students (DES, $21 \pm 2$ yrs.) were accompanied over their $\approx 5$ years of college. Changes of physical activity and exercise were assessed by dedicated questionnaires and interviews. Metabolic syndrome (MetS)-Z-Score based on the NCEP ATP III definition of the MetS, abdominal fat (\%) as assessed by Dual Energy X-Ray Absorptiometry (DXA) and cardiorespiratory fitness (CRF) as assessed by stepwise bicycle ergometry determined the cardiometabolic risk at baseline and 5 year follow-up. 
Results: 67 percent of the subjects initially included completed the follow-up-assessments. During the 5-year study period physical activity non-significantly increased in both groups $(5-10 \%, P>$.15). Indices of exercise increased significantly in the SPS group $(P<.001)$ and decreased significantly $(P<.001)$ in the DES group. Group differences were significant $(P \leq .047)$ for all study endpoints with more favorable changes among the SPS group for MetS-Z-Score $(-0.25 \pm 0.56, P=.367$ vs. DES: $1.44 \pm 0.78, P<.001)$, abdominal body fat $(1.9 \pm 12.8 \%, P=.539$ vs. $10.9 \pm 21.8 \%, P=.004)$ and $\mathrm{CRF}$ $(1.6 \pm 2.9 \%, P=.076$ vs. $-3.3 \pm 4.1 \%, P=.004)$.

Conclusion: Reductions of exercise volume and particularly exercise intensity caused by occupational factors during the college years may be the most prominent risk factor for cardiometabolic diseases in young adults. Compensatory exercise is thus highly relevant and may offset an otherwise unhealthy lifestyle.

Keywords: Metabolic syndrome; abdominal body fat; cardiorespiratory fitness; students; university.

\section{INTRODUCTION}

Transition to early adulthood with its pronounced changes of lifestyle can severely challenge a subject's health status [1,2]. Indeed, the move from (high) school to university, traineeship or job especially along with moving away from home may lead to unhealthy changes of job demands, stress, drug abuse, eating and sleeping pattern and general problems in coping with the new, unfamiliar setting [3-5]. With respect to cardiometabolic risk factors, the 5.5-times higher weight gain during the "freshmen period" compared with the general population [6], which can be largely attributed to increases of fat mass [7] may be the most prominent consequence of this new situation. In parallel, physical activity as a protective factor declines above average during this period of life [5,8], which may also be a direct outcome of the new, unfamiliar setting [9]. In summary, these adverse changes in fitness, fatness and lifestyle are causative contributors to the metabolic syndrome as an important basis of cardiovascular disease [10]. Cardiovascular diseases however still represents the leading cause of death, at least in the US [11]. Maintaining or increasing the amount of sport and/or physical exercise may be the most effective tool in combatting cardiometabolic risk factors in this period of life. The aim of this project was to determine the effect of exercise on the development of the metabolic syndrome (MetS), abdominal fatness and cardiorespiratory fitness (CRF). In order to achieve this goal we accompanied two cohorts of students with fundamentally different exercise patterns (sports vs. dentistry students), but comparable setting and situation during their study course of $\approx 5$ years. Our primary hypothesis was that 5-year changes of (a) MetS-Z-Score, (b) abdominal fat mass and (c) CRF differ significantly between sports students (SPS) and dentistry students
(DES) with more favorable changes among the SPS.

\section{METHODS}

This study was a 5-year comparative "intervention" study that focused on the effect of work-related activity and exercise on health risk factors in young adults. The study was conducted from May 2007 through December 2013 by the Institute of Medical Physics, Friedrich Alexander-University ErlangenNuremberg (FAU), Germany. The study protocol was approved by the ethics committee of the University of Erlangen (Ethik Antrag 3674) and the Bundesamt für Strahlenschutz (Z5-22462/22007-041). All the study participants signed a written informed consent. The study was fully registered under www.clinicaltrials.gov (NCT00521235).

\subsection{Participants}

One hundred study starters each of the disciplines "dentistry" and "sport sciences" selected by computer-generated random lists based on lists of (FAU) study starters of the years 2007 and 2008 were contacted by mail. 157 subjects responded and were further informed in detail with respect to the aims, risks and benefits of the study. 44 subjects were unwilling to participate or were ineligible due to our exclusion criteria of (a) intended change of study program or study location, (b) age $\geq 30$ years (c) diseases/medication affecting body composition, (d) pregnancy. Finally 114 eligible subjects (DES: $n=61$ vs. SPS: $n=53$ ) were included in the study (Fig. 1). Table 1 gives baseline characteristics of both groups.

With the exception of body fat, (both genders) and waist circumference (women only) 
anthropometric, dietary and CVD-risk baseline values given in Table 1 did not vary between male SPS vs. DES and female SPS vs. DES. Baseline physical activity did not differ between the groups, however exercise parameters were significantly more favorable in the SPS group (Table 1).

\section{2 "Intervention"}

Study contents and volume of both study programs that could be considered as the "intervention" were exactly prescribed in the corresponding curricula for dentistry and sport sciences. Further, subjects were assessed immediately after the start of their study program ( $\leq 4$ weeks) and during the last semester of their study program as part of the "intervention" approach we adopted. Both study protocols were described in detail in a preceding article [12], thus only a brief characterization will be given here.

The study of dentistry is considered as a comprehensive and stressful study program. However, physical activity during this occupation is low due to the rather immobile sitting and (less frequent) standing positions [13]. Altogether, the regular study period for dentistry is 11 semesters with an average of $32-36 \mathrm{~h} /$ week during the semester. Since dentistry internships and practical work were obligatory and the key examinations took part during the 2-month "semester breaks", the occupational workload and stress remained at a high level. Although individual time of preparation was not listed in the study protocol, responses in the questionnaires provided after the $4^{\text {th }}$ and $9^{\text {th }}$ semesters put the weekly workload directly or indirectly related to the dentistry course at an average of $32 \pm 9$ and $37 \pm 12 \mathrm{~h} /$ week respectively.

Sports students aiming to qualify as secondaryschool teachers in Germany, on the other hand, have to be extremely physically active. Altogether, 1,050 obligatory hours of practical sport are required during the 9-semester study curriculum, but preparation for sports training, corresponding tests and leisure time sports activity increase this amount up to $11.9 \pm 2.4 \mathrm{~h} / \mathrm{w}$. (range: 8-17 h/w.). Obligatory core and facultative disciplines included all types of exercises (i.e. gymnastics, track and field athletics, swimming, dancing, team and individual ball games, skiing, water sports and martial arts). During the semester breaks,

Table 1. Baseline characteristics of male and female sport (SPS) and dentistry students (DES)

\begin{tabular}{|c|c|c|c|c|}
\hline Variable (at baseline) & Sport-Sciel & e (SPS) & Dentistr & DES) \\
\hline Gender & Female $n=29$ & Male $n=24$ & Female $n=33$ & Male $n=28$ \\
\hline Age [years] & $20.1 \pm 2.1$ & $20.6 \pm 1.9$ & $20.5 \pm 2.5$ & $21.1 \pm 3.1$ \\
\hline Body height $[\mathrm{cm}]$ & $167.5 \pm 6.4$ & $181.0 \pm 5.5$ & $169.2 \pm 7.1$ & $181.3 \pm 7.0$ \\
\hline Body weight $[\mathrm{kg}]$ & $62.5 \pm 7.4$ & $72.8 \pm 6.6$ & $59.8 \pm 6.4$ & $75.5 \pm 8.7$ \\
\hline European descent [\%] & 97 & 92 & 97 & 93 \\
\hline Al [hours/week] ${ }^{a}$ & $30 \pm 10$ & $28 \pm 9$ & $32 \pm 9$ & $27 \pm 11$ \\
\hline $\mathrm{EI}[\mathrm{min} / \text { week] }]^{\mathrm{b},}$ & $221 \pm 115$ & $270 \pm 126$ & $109 \pm 65$ & $123 \pm 71$ \\
\hline $\mathrm{IEI}_{\text {aer }}[\mathrm{min} / \text { week/intensity }]^{\mathrm{c},{ }^{*}}$ & $241 \pm 124$ & $328 \pm 148$ & $112 \pm 88$ & $131 \pm 92$ \\
\hline $\mathrm{IEI}_{\text {res }}[\mathrm{min} / \text { week/intensity }]^{\mathrm{d},{ }^{*}}$ & $85 \pm 57$ & $109 \pm 52$ & $37 \pm 27$ & $23 \pm 17$ \\
\hline Active in Competitions [\%] ${ }^{*}$ & 100 & 100 & 21 & 25 \\
\hline $\mathrm{VO}_{2}$ peak $[\mathrm{ml} / \mathrm{min} / \mathrm{kg}]^{\mathrm{e}^{\pi}}$ & $46.1 \pm 4.2$ & $57.4 \pm 5.5$ & $42.2 \pm 5.8$ & $52.0 \pm 8.8$ \\
\hline Total body fat (DXA) [\%] ${ }^{\star}$ & $23.8 \pm 4.2$ & $12.5 \pm 3.1$ & $26.3 \pm 3.9$ & $16.7 \pm 4.0$ \\
\hline Waist circumference $[\mathrm{cm}]^{\star *}$ & $76.4 \pm 4.4$ & $79.5 \pm 3.1$ & $72.6 \pm 5.6$ & $81.0 \pm 6.6$ \\
\hline $\mathrm{MAP}^{6}[\mathrm{mmHG}]$ & $93.0 \pm 6.9$ & $99.3 \pm 6.1$ & $96.1 \pm 7.2$ & $99.0 \pm 9.5$ \\
\hline Glucose [mg/dl] & $85.0 \pm 5.8$ & $86.5 \pm 6.3$ & $82.9 \pm 7.6$ & $89.9 \pm 8.9$ \\
\hline Triglyzerides [mg/dl] & $91.7 \pm 36.2$ & $88.6 \pm 42.5$ & $109.3 \pm 53.4$ & $99.2 \pm 46.4$ \\
\hline HDL-C [mg/dl] & $68.0 \pm 14.4$ & $55.7 \pm 10.5$ & $71.6 \pm 16.1$ & $56.1 \pm 11.5$ \\
\hline Energy uptake [kcal/d] & $2301 \pm 591$ & $2659 \pm 612$ & $2119 \pm 408$ & $2714 \pm 643$ \\
\hline Carb/Prot./Fat/Alcohol [\%] $]^{\dagger}$ & $59 / 18 / 22 / 1$ & $58 / 20 / 18 / 4$ & $65 / 14 / 20 / 1$ & $57 / 18 / 21 / 4$ \\
\hline Alcohol intake $[\mathrm{g} / \mathrm{d}]$ & $3.3 \pm 2.4$ & $13.9 \pm 7.4$ & $4.1 \pm 3.6$ & $14.4 \pm 9.0$ \\
\hline
\end{tabular}




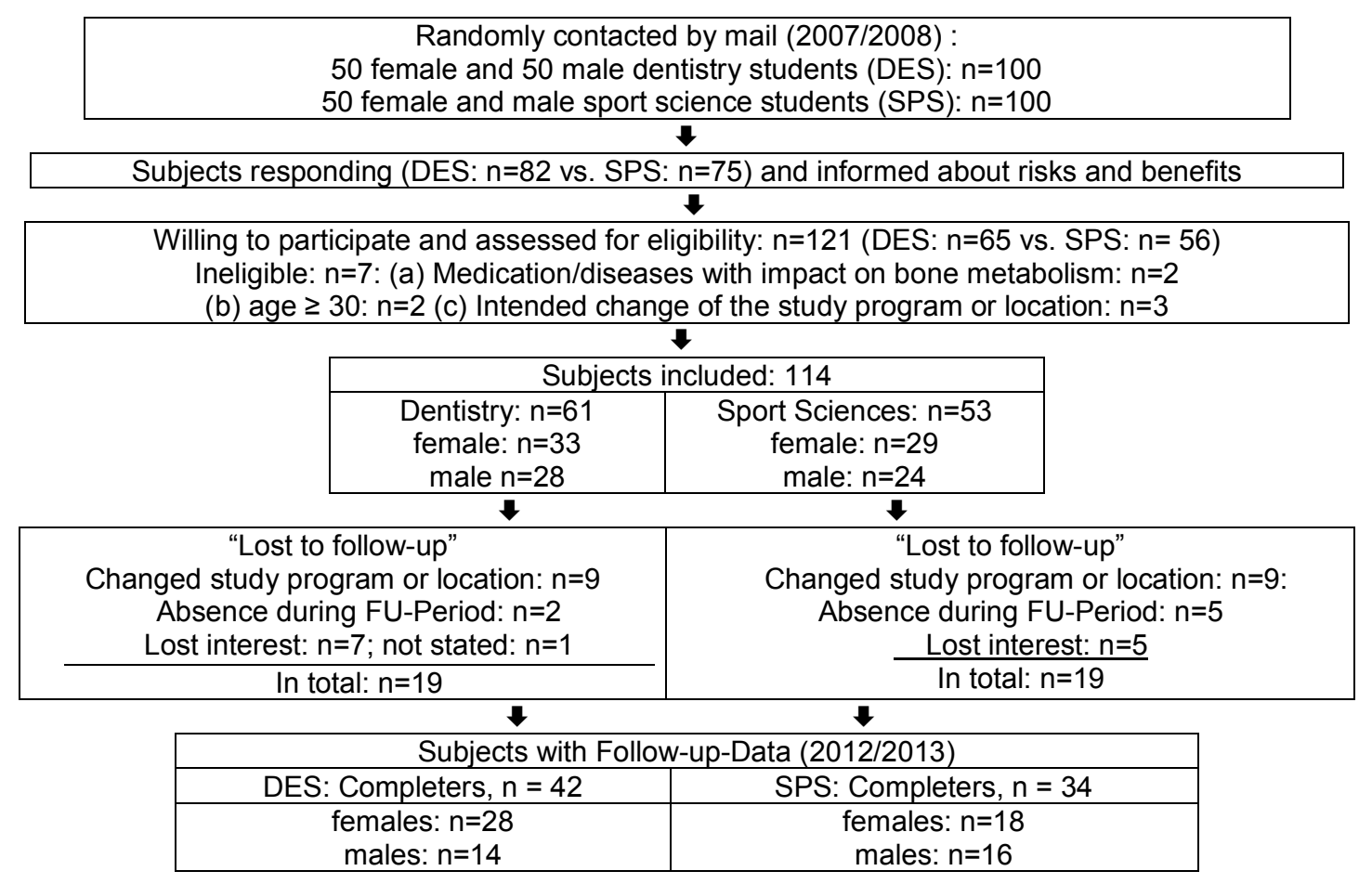

Fig. 1. "Flow-chart" of the study

the total amount of exercise remained high (i.e. $6 \pm 3 \mathrm{~h} / \mathrm{w}$.) due to preparation for tests and increased leisure time sports activity. Thus, the "study intervention", which took the subjects levels of exercise that were already high before they embarked on their degree course the (Table. 1), can be considered as an optimal prevention program with respects to metabolic and cardiovascular risk factors.

Both cohorts further reported comparable additional general physical activity due to paid work outside the study program that averaged $9 \pm 6 \mathrm{~h} /$ week.

\subsection{Outcome}

The primary study outcome addressed in this article was the change of the Metabolic Syndrome Z-Score (MetS-Z-Score) according to Johnson et al. [14] from baseline to final followup (5 years). Secondary endpoints were corresponding changes of abdominal fat (AF) as assessed by Dual Energy $x$-Ray absorptiometry (DXA) and cardiorespiratory fitness (CRF) as assessed by ergo-spirometry (Viasys, Conshohocken, USA).

\subsubsection{Testing procedures}

Baseline tests were performed $\leq 6$ weeks after the start of the study program. However due to the different study duration (SPS: 9 vs. DES: 11 semester), final follow-up assessment of the SPS group was consistently after their final semester or during the first months of their school internship $(4.7 \pm 0.4 \mathrm{yrs})$, while dentist students were finally tested during the semester break between the $9^{\text {th }}$ and $10^{\text {th }}$ semesters $(4.8 \pm 0.5 \mathrm{yrs})$.

Strong emphasis was given to assessing subjects at the same time of the day $( \pm 2 \mathrm{~h})$ in a fixed order and by the same researcher. Assessments were carried out in a blinded fashion, i.e. researchers were not informed about the status of the subjects (DES or SPS).

\subsubsection{Anthropometry}

Height was determined with a stadiometer and weight was measured with minimal clothing on digital scales. Waist circumference was determined as the minimum circumference between the distal end of the rib cage and the top of the iliac crest along the midaxillary line. Body composition was assessed with DualEnergy X-Ray Absorptiometry (DXA) (QDR 4500, discovery upgrade, Hologic Inc., Bedford, USA) at baseline and after $4.8 \pm 0.5$ years, using the whole body standard protocol specified by the manufacturer. Region of interest (ROI) for abdominal body fat was determined between the 
lower edge of the $12^{\text {th }}$ rib and the upper edge of the iliac crest.

\subsubsection{Metabolic syndrome}

MetS-Z-Score was calculated according to the formula proposed by Johnson et al. [14] based on the NCEP-ATP III criteria of the MetS [15]. Following Johnson et al. [14], the NCEP ATP-III gender specific cut-point and the corresponding baseline standard deviation (SD) of the corresponding cohort (DES or SPS) were used for each parameter of the individual data. In detail, the MetS-Z-Score was separately calculated for males and females using: [(40 $(\mathrm{m})$ or 50 (f)-HDL-C)/SD HDL-C] + [(TriGly-180)/SD TriGly $]+[($ Glucose-100)/SD Glucose $]+[(\mathrm{WC}-$ $88(\mathrm{f})$ or $102(\mathrm{~m}) / \mathrm{SD} \mathrm{WC}]+[($ Mean arterial pressure (MAP)-100)/SD MAP]. SD of each component was calculated separately for baseline and final follow-up.

\subsubsection{Questionnaires}

Detailed baseline questionnaires were completed by all the participants. Strong emphasis was placed on accurately determining physical activity, sports and exercise levels and their corresponding changes during the study course. Specific questionnaires and personal interviews [16] determined the history, type, volume and self-rated intensity of physical activity, sports and exercise. Based on this questionnaire, three sports scientists (WK, MB, SvS) calculated several indices using the Delphi technique [17]. Four indices were selected for the present research questions: (1) the activity intensity index (Al), as a summary of total physical activity (h/week) under consideration of the type and intensity of this activity, rated on a 7-item scale; (2) the total exercise index (El, in min/week) that simply characterizes total volume of sports and exercise per week (i.e. weekly frequency $x$ duration per session). Further, (3) El was structured according to the type of sports and exercise [(either aerobic $\left(\mathrm{El}_{\mathrm{aer}}\right)$ or resistance type $\left(\mathrm{El}_{\text {res }}\right)$ exercise (or neither) in $\mathrm{min} /$ week]. Finally, (4) an exercise intensity index for both types of exercise $\left(\mathrm{IEI}_{\text {aer }} ; \quad \mathrm{IEI} \mathrm{I}_{\text {res }}\right)$ was calculated by multiplying $\mathrm{El}_{\text {aer/res }}(\mathrm{min} /$ week) x 1 (low), x 2 (moderate), or $\times 3$ (high intensity).

In addition, follow-up questionnaires and structured interviews were completed in order to control changes of parameters that may confound our results (e.g. lifestyle, medication, diseases). 4-day dietary protocols were completed at baseline, after the $4^{\text {th }}$ semester and during the final FU-assessment. Food consumed was analyzed using the "Freiburger Nutrition Protocol" (nutri-science, Hausach, Germany).

\subsection{Statistics}

The sample size calculation was based on the primary endpoint "Metabolic Syndrome Z-Score" [14]. In order to detect a group difference ( $\delta$-SPS vs. $\delta$-DES) of $1 \pm 1.5$ (Z-Score) (Type I: $5 \%$, Type II: $80 \%$ ) given an anticipated drop-out rate of about one third, 36 subjects/group were required.

Baseline values are given as means with standard deviations $(\mathrm{MV} \pm \mathrm{SD})$. Differences between baseline and follow-up per group were reported as absolute (Table 2) or percentage changes (text). Due to the different proportion of males and females per group, baseline values were adjusted accordingly. Between-group differences are given as absolute difference with 95\% confidence interval (Table 2). Paired and unpaired Welch-t-tests were used as appropriate, where all the tests were 2-sided using a significance level of 0.05 . Effect sizes (ES) based on the absolute difference ( \pm standard deviation) between baseline and follow-up in the SPS and DES were calculated using Cohen's d (20). SPSS 21.0 (SPSS Inc, Chicago, IL) was used except for the ITT-analysis.

The ITT-analysis was performed using the statistics software R ( $R$ Development Core Team Vienna, Austria) in combination with multiple imputation by Amelia II [18]. Following the recommendations of Honaker et al. [18] and Graham et al. [19] the full data set was used for multiple imputation, with imputation being repeated 50 times. In addition, the approach of Barnard et al. [20] was used to compute mean, SD (combination of within- and betweenimputation variance) and $P$ values (t-distribution with adjusted degrees of freedom). In all cases, the results obtained were in very good agreement with the respective results determined with the approach of Steele et al. [21].

\section{RESULTS}

Fig. 1 shows the flow chart of the study. In both study groups, nineteen subjects each were lost to follow-up: Eighteen subjects changed their study program and/or study location (outside southern Germany). Three SPS had to abandon their sport study program due to musculoskeletal injuries. Seven subjects were absent during the 
FU-assessment period, 12 subjects lost interest and/or were unwilling to perform the final FU. One subject did not give a reason for his withdrawal.

\subsection{Changes of Dietary Intake, Alcohol Consumption and Smoking}

No relevant changes of dietary intake were observed in the SPS-or DES-group from baseline to follow-up. Energy uptake increased slightly by $66 \pm 533 \mathrm{kcal}$ in the SPS and $41 \pm 469 \mathrm{kcal}$ in the DES with no relevant changes of the proportion of macronutrients. Alcohol consumption increased slightly in the male SPS and DES (4\% and $6 \%$ respectively) but doubled $(P=.001)$ in the female cohort to $7.6 \pm 6.1$ (SPS) and $7.1 \pm 6.1 \mathrm{~g} / \mathrm{d}$ (DES). Only $18 \%$ of the DES and $6 \%$ of the SPS smoked. Smoking habits of the DES and SPS groups did not change significantly during the study period.

\subsection{Changes of Lifestyle, Physical Activity, Sports and Exercise}

Due to obligatory military or civil service, preceding internship or employment only $36 \%$ of the SPS and $38 \%$ of the DES changed immediately from high school to university (females SPS: $56 \%$, DES: $52 \% ; P<.001$ vs. males).

Based on comparable baseline data (Table. 1), the activity intensity index (Al) increased nonsignificantly $(P>.15)$ by $5-10 \%$ in both groups with no significant group differences $(P=.689)$ and no differences between genders. Changes were related to earning a livelihood.

Independent of gender, baseline values for the three exercise indices calculated were significantly higher $(P=.001)$ in the sports compared with the dentistry students, with the most impressive differences for the intensity exercise indices (IEI) for resistance and aerobic exercise type.

"Non-occupational" El (i.e. leisure time-sports activities) was maintained in the SPS $(-2 \pm 13 \%$, $P=.359$ ) but decreased significantly in the DES group $(-34 \pm 22 \%, P<.001)$. Differences between the groups were significant $(P<.001)$. Moreover, the number of subjects exercising at least 2 sessions a week decreased by $32 \%$ in the DES group. The most impressive reduction among the DES was determined for the intensity exercise indices (IEI). Both resistance and aerobic IEI decreased by $-38 \pm 22 \%$ and $-41 \pm 32 \%(P<.001)$, whereas these parameters were maintained in the SPS group. However, these values refer to leisure time exercise with respect to the SPS group, thus one has to add the high volume and intensity of "occupational" exercise arising from the study program given above.

\subsection{Primary and Secondary Endpoints}

After 5 years, trends for female versus male DES or SPS peers were nearly identical, so a combined analysis of male and female DES vs. male and female SPS was performed.

Results of the ITT-analysis are listed in Table. 2. Based on identical baseline values $(P=.972)$, the MetS-Z-Score was maintained in the SPS $(P=.367)$ and deteriorated significantly in the DES $(P<.001)$ group. Differences with respect to intragroup changes were significant $(P<.001$; $\left.d^{`}=1.04\right)$.

Table 2. Baseline, intragroup changes and intergroup differences for the metabolic syndrome Z-Score (MetS-Z-Score) according to Johnson et al. [14], abdominal fat and cardiorespiratory fitness. data assessed by "intention to treat" analysis

\begin{tabular}{|c|c|c|c|c|c|}
\hline & SPS $n=51$ & DES $n=63$ & $\begin{array}{l}\text { Absolute difference } \\
\mathrm{MV}(95 \% \mathrm{Cl})\end{array}$ & $P$ & (d) \\
\hline $\begin{array}{l}\text { MetS-Z-Score } \\
\text { Baseline } \\
\text { Difference }\end{array}$ & $\begin{array}{l}-8.08 \pm 2.12 \\
-0.25 \pm 0.56 \\
\text { n.s. }\end{array}$ & $\begin{array}{l}-8.09 \pm 2.72 \\
1.44 \pm 0.78^{\star \star * *}\end{array}$ & 1.69 (0.89 to 2.49$)$ & $\begin{array}{l}.972 \\
<.001\end{array}$ & $----{ }^{--5}$ \\
\hline $\begin{array}{l}\text { Abdominal fat [\%] } \\
\text { Baseline } \\
\text { Difference }\end{array}$ & $\begin{array}{l}14.50 \pm 4.96 \\
0.28 \pm 1.43^{\text {n.s. }}\end{array}$ & $\begin{array}{l}17.01 \pm 4.88 \\
1.86 \pm 2.86^{* *}\end{array}$ & 1.59 (0.02 to 3.15$)$ & $\begin{array}{l}.009 \\
.047\end{array}$ & $\begin{array}{l}-.-- \\
.70\end{array}$ \\
\hline $\begin{array}{l}\text { Cardiorespiratory fit } \\
\text { Baseline } \\
\text { Difference }\end{array}$ & $\begin{array}{c}s[\mathrm{ml} / \mathrm{min} / \mathrm{kg}] \\
51.23 \pm 7.40 \\
081+140^{\text {n.s. }}\end{array}$ & $\begin{array}{l}46.62 \pm 7.59 \\
-1.53+181^{* *}\end{array}$ & $255(100$ to 367$)$ & $\begin{array}{l}.001 \\
<001\end{array}$ & --- \\
\hline
\end{tabular}


Abdominal body fat [\%] differed significantly between SPS and DES at baseline $(P=.001)$. After 5 years of university, abdominal fat changes were significantly higher $(P=.047$, $\left.\mathrm{d}^{\prime}=.70\right)$ in the DES $(10.9 \pm 21.8 \%, P=.004)$ compared with the SPS $(1.9 \pm 12.8 \%, P=.539)$ (Table 2).

Baseline CRF varied significantly between SPS and DES $(51.2 \pm 7.4$ vs. $46.6 \pm 7.6 \mathrm{ml} / \mathrm{kg} / \mathrm{min}$, $P<.001)$. CRF further increased by $1.6 \pm 2.9 \%$ in the SPS $(P=.076)$ and decreased significantly in the DES $(3.3 \pm 4.1 \%, P=.003)$. The corresponding intergroup difference based on absolute changes was significant $\left(P<.001, d^{`}=1.45\right)$ (Table 2$)$.

Thus, hypotheses (a)-(c) can be fully confirmed.

\section{DISCUSSION}

The pronounced life style changes during young adulthood may particularly impact general physical activity $[1,5,22]$ and, to an even greater degree, sports and physical exercise [23]. Reviewing the literature (e.g. [1,5,22]), most studies reported significant reductions of physical activity related to the new occupational situation (i.e. military/civilian service, apprenticeship, job, university). However, depending on their magnitude, reductions of exercise volume and/or intensity during young adulthood may result in severe negative changes in various health parameters [24]. Therefore, the main aim of this contribution was to determine the effect of sports and exercise changes, and the corresponding impact on cardiometabolic risk factors (MetS-Zscore and abdominal fat) and cardiorespiratory fitness (CRF).

With respect to physical activity, a slight increase was observed in both groups. This apparently unexpected (i.e. $[5,22,25,26]$ ) finding becomes more plausible after looking behind this "umbrella term". For example, German data [23] indicate that the number of subjects who say they perform $\geq 30 \mathrm{~min} / \mathrm{d}$ moderate to vigorous physical tasks per day does not vary relevantly between the age of 18 to 29 years. At the same time however, participation in sports and exercise of $\geq 2$ hours/w. during this period decreased by 25$30 \%$ in both genders [23], which is much in line with our DES data. Addressing intensity, a significant decrease of aerobic (IElaer) and resistance (IEIres) exercise intensity along with a reduced participation in competitions and tournaments in the DES group was observed. This shift to less vigorous exercise may have been even more harmful with respect to MetSparameters [27,28], abdominal fat [29] and $\mathrm{CRF} / \mathrm{VO}_{2}$ max $[28,30]$ than the pure reduction of exercise volume alone.

In summary, the results suggest that changes of sport and exercise habits, induced by the new occupational situation of the young adult, severely affect cardiometabolic risk factors and fitness. One may argue that other study-or setting-related factors may have made relevant contributions to this result. However, we were unable to detect significant longitudinal group differences for parameters other than exercise. To our surprise, with one exception (alcohol consumption), we did not observe any relevant changes of confounding parameters (e.g. dietary intake, sun exposure, smoking and sleeping habits), which were basically in a totally normal range $[31,32]$. We are aware that this finding is not in accordance with US data that reported a variety of unhealthy changes during college/university years (e.g. [3,5]). We attribute this differing outcome primarily to the fact that the subjects of the present study were older, predominately did not directly move from parental home to university and may have been more life experienced and rational.

With respect to the corresponding dose response interactions it is of interest that even a highly significant increment of the already outstanding baseline exercise habits of the SPS did not further improve MetS $(P=.367)$, abdominal fatness $(P=.539)$ or $\mathrm{CRF}(P=.076)$, suggesting a ceiling effect for this cohort. We confirmed this appraisal by a corresponding subanalysis (not given here), that did not detect significant differences $(P \geq .381)$ for the parameters given above among SPS in the highest versus their peers in lowest tercile of exercise changes.

Much more relevant, however, is the significant aggravation of parameters closely related to cardiometabolic diseases [10,33-35] in the DES. Looking behind the covariates, the MetS-Z-Score change for this group can be partially attributed to the $3.9 \pm 5.8 \mathrm{~cm}(P<.001)$ increase of waist circumference which was confirmed by the $17 \%$ gain of abdominal fat mass (Table. 2) as assessed by DXA. Other unfavorable changes were determined for triglycerides $(+12 \pm 27 \%$, $P=.008)$ and MAP $(6.3 \pm 8.0 \%, P=.001)$, whereas none of the parameters constituting the MetS, changed significantly in the SPS group. In contrast to the SPS-group, we determined significant differences (CRF: $P=.001$ to MetS-Z- 
Score: $P=.015)$ between DES in the highest tercile of exercise changes compared with the tercile that reported no or slight changes only (data not given).

Some features of the present study may limit the evidence that reductions of sports/exercise volume and/or intensity were primarily responsible for the aggravation of cardiometabolic risk factors and CRF in young adults. (a) Basically the study design may complicate the proper identification of causal effects. Comprehensively we were not able to randomize candidates to the DES or SPS-study protocol nor to prescribe the study curriculum. On the other hand, one should consider that this "observational prospective study" exhibits some strong interventional characteristics. Firstly, the study programs can be considered as extremely pronounced interventions. Further, the study programs are exactly prescribed in the study program and mandatory for all students, thus, contrary to most interventional studies, results can hardly be confounded by subject (non-) compliance. Finally, in order to completely determine the impact of the "intervention", subjects were directly assessed after study program start. (b) One may argue that a selection bias confound our results. Although there may be some rare cohorts that may be destined to much or less weight/fat gain and cardiometabolic diseases independent of changes of physically activity and exercise, it is not likely that the large cohort of German sports students or vice versa the cohort of dentistry students were exceptionally predisposed in this respect. (c) Although strong emphasis was placed on detecting possible confounders, some relevant changes may have escaped our attention. (d) Although the rate of males to females included in the final analysis considerably differ (DES: 1:2 vs. SPS: 1:1.1) between the groups, this do not affect our result due to nearly identical trends in both genders of the given cohort.

\section{CONCLUSION}

We conclude that the most deleterious effect on cardiometabolic parameters in young adults was causally related to the severe decrease in sports and exercise volume and intensity observed during this period. Consequently, the subjects' maintenance of exercise habits, ideally supported by the arrangements at their place of study, work, etc. so as to provide adequate possibilities and encouragement for sport and exercise, is of high relevance $[33,35,36]$ and may compensate for an otherwise unhealthy lifestyle. In detail, time-effective aerobic and resistance HIT-exercise protocols $[37,38]$ may be the most feasible and efficient option for realizing this aim.

\section{COMPETING INTERESTS}

Authors have declared that no competing interests exist.

\section{REFERENCES}

1. Ferrara CM. The college experience: Physical activity, nutrition and implications for intervention and future research. JEP Online. 2009;12:23-35.

2. Small M, Bailey-Davis L, Morgan N, Maggs J. Changes in eating and physical activity behaviors across seven semesters of college: Living on or off campus matters. Health Educ. Behav. 2013;40:435-441.

3. Racette SB, Deusinger SS, Strube MJ, Highstein GR, Deusinger RH. Changes in weight and health behaviors from freshman through senior year of college. J. Nutr Educ. Behav. 2008;40:39-42.

4. Steptoe A, Wardle J. Health behaviour, risk awareness and emotional well-being in students from Eastern Europe and Western Europe. Soc Sci Med. 2001;53:1621-1630.

5. Kwan MY, Cairney J, Faulkner GE, Pullenayegum EE. Physical activity and other health-risk behaviors during the transition into early adulthood: A longitudinal cohort study. Am J. Prev Med. 2012;42:14-20.

6. Mihalopoulos NL, Auinger P, Klein JD. The Freshman 15: Is it real? J. Am Coll Health. 2008;56:531-533.

7. Gropper SS, Simmons KP, Connell LJ, Ulrich PV. Changes in body weight, composition and shape: A 4-year study of college students. Appl Physiol Nutr Metab. 2012;37:1118-1123.

8. Ortega FB, Konstabel K, Pasquali E, Ruiz JR, Hurtig-Wennlof A, Maestu J, et al. Objectively measured physical activity and sedentary time during childhood, adolescence and young adulthood: A cohort study. PLoS One. 2013;8:e60871.

9. Fransson El, Heikkila K, Nyberg ST, Zins $M$, Westerlund $H$, Westerholm $P$, et al. Job strain as a risk factor for leisure-time physical inactivity: An individual-participant meta-analysis of up to 170,000 men and 
women: The IPD-Work consortium. Am J. Epidemiol. 2012;176:1078-1089.

10. Wilson PW, Kannel WB, Silbershatz $H$, D'Agostino RB. Clustering of metabolic factors and coronary heart disease. Arch Intern Med. 1999;159:1104-1109.

11. Santulli G. Epidemiology of Cardiovascular Disease in the $21^{\text {st }}$ Century: Updated numbers and updated facts. JCvD. 2013; 1:1-2.

12. Kemmler $W$, Bebenek $M$, von Stengel $S$, Bauer J. Peak-bone-mass development in young adults: Effects of study program related levels of occupational and leisure time physical activity and exercise. A prospective 5-year study. Osteoporos Int; 2014. (Online first).

DOI:10.1007/s00198-00014-02918-00198.

13. Kierklo A, Kobus A, Jaworska M, Botulinski B. Work-related musculoskeletal disorders among dentists-a questionnaire survey. Ann Agric Environ Med. 2011;18: 79-84.

14. Johnson JL, Slentz CA, Houmard JA, Samsa GP, Duscha BD, Aiken LB, et al. Exercise training amount and intensity effects on metabolic syndrome (from Studies of a Targeted risk reduction intervention through defined exercise). Am J. Cardiol. 2007;100:1759-1766.

15. Program NCE. Executive summary of the third report of the National Cholesterol Education Program (NCEP) Expert panel on detection, evaluation and treatment of High blood cholesterol in adults (adult treatment panel III). JAMA. 2001;285: 2486-2497.

16. Kemmler W, Weineck J, Kalender WA, Engelke K. The effect of habitual physical activity, non-athletic exercise, muscle strength and VO2max on bone mineral density is rather low in early postmenopausal osteopenic women. J. Musculoskelet Neuronal Interact. 2004;4: 325-334.

17. Hasson F, Keeney S, McKenna $H$. Research guidelines for the Delphi survey technique. J. Adv Nurs. 2000;32:10081015.

18. Honaker J, King G, Blackwell M. Amelia II: A program for missing data JSS. 2011;45: 1-47.

19. Graham JW, Olchowski AE, Gilreath TD. How many imputations are really needed? Some practical clarifications of multiple imputation theory. Prev Sci. 2007;8:206213.
20. Barnard J, Rubin DB. Small-sample degrees of freedom with multiple imputation. Biometrika. 1999;86:948-955.

21. Steele RJ, Wang N, Raftery AE. Inference from Multiple imputation for missing data using mixtures of normals. Stat Methodol. 2010;7:351-364.

22. Knuth $A G$, Hallal PC. Temporal trends in physical activity: A systematic review. J. Phys Act Health. 2009;6:548-559.

23. Rütten $A$, Abu-Omar K, Lampert $T$, Ziese T. Körperliche Aktivität [ physical activity]. report. in: Robert-Koch-Institut, ed. Gesundheitsberichterstattung des Bundes. (Berlin: Statistisches Bundesamt); 2005.

24. Rangul $V$, Bauman $A$, Holmen $T L$, Midthjell K. Is physical activity maintenance from adolescence to young adulthood associated with reduced CVD risk factors, improved mental health and satisfaction with life: The HUNT Study, Norway. Int J. Behav Nutr Phys Act. 2012; 9:144.

25. Anderssen N, Jacobs DR. Jr, Sidney S, Bild DE, Sternfeld B, Slattery ML, et al. Change and secular trends in physical activity patterns in young adults: A sevenyear longitudinal follow-up in the Coronary Artery Risk Development in Young Adults Study (CARDIA). Am J. Epidemiol. 1996; 143:351-362.

26. Gordon-Larsen P, Nelson MC, Popkin BM. Longitudinal physical activity and sedentary behavior trends: Adolescence to adulthood. Am J. Prev Med. 2004;27:277283.

27. Kessler HS, Sisson SB, Short KR. The potential for high-intensity interval training to reduce cardiometabolic disease risk. Sports Med. 2012;42:489-509.

28. Tjonna AE, Lee SJ, Rognmo O, Stolen TO, Bye A, Haram PM, et al. Aerobic interval training versus continuous moderate exercise as a treatment for the metabolic syndrome: A pilot study. Circulation. 2008;118:346-354.

29. Vissers D, Hens W, Taeymans J, Baeyens JP, Poortmans J, Van Gaal L. The effect of exercise on visceral adipose tissue in overweight adults: A systematic review and meta-analysis. PLoS One. 2013;8:e56415.

30. Helgerud J, Hoydal K, Wang E, Karlsen T, Berg $P$, Bjerkaas $M$, et al. Aerobic highintensity intervals improve VO2max more than moderate training. Med Sci Sports Exerc. 2007;39:665-671. 
31. Germany Society for Nutrition. Dietary Reference values. Neuer Umschau buchverlag, Bremen; 2012.

32. Statistisches-bundesamt. Gesundheit in Deutschland [health in Germany]. Gesundheitsberichterstattung des Bundes. Berlin; 2006.

33. Carnethon MR, Gidding SS, Nehgme R, Sidney S, Jacobs DR. Jr, Liu K. Cardiorespiratory fitness in young adulthood and the development of cardiovascular disease risk factors. JAMA. 2003;290:3092-3100.

34. Despres JP, Pouliot MC, Moorjani S, Nadeau A, Tremblay A, Lupien PJ, et al. Loss of abdominal fat and metabolic response to exercise training in obese women. Am J. Physiol. 1991;261:E159167.
35. Santulli G, Ciccarelli M, Trimarco B, laccarino G. Physical activity ameliorates cardiovascular health in elderly subjects: The functional role of the beta adrenergic system. Front Physiol. 2013;4:209.

36. Gropper SS, Simmons KP, Connell LJ, Ulrich PV. Weight and Body Composition Changes during the First Three Years of College. J. Obes; 2012.

37. Bird SR, Hawley JA. Exercise and type 2 diabetes: New prescription for an old problem. Maturitas. 2012;72:311-316.

38. Gibala MJ. High-intensity interval training: A time-efficient strategy for health promotion? Curr Sports Med Rep. 2007;6: 211-213.

(c) 2015 Kemmler et al.; This is an Open Access article distributed under the terms of the Creative Commons Attribution License (http://creativecommons.org/licenses/by/4.0), which permits unrestricted use, distribution and reproduction in any medium, provided the original work is properly cited.

Peer-review history:

The peer review history for this paper can be accessed here: http://www.sciencedomain.org/review-history.php?iid=1118\&id=12\&aid=9162 